\title{
Conformations of tissue plasminogen activator (tPA) orchestrate neuronal survival by a crosstalk between EGFR and NMDAR
}

\author{
T Bertrand ${ }^{1,2}$, F Lesept ${ }^{1,2}$, A Chevilley ${ }^{1}$, S Lenoir ${ }^{1}$, M Aimable ${ }^{1}$, A Briens ${ }^{1}$, Y Hommet ${ }^{1}$, I Bardou ${ }^{1}$, J Parcq ${ }^{1}$ and D Vivien ${ }^{\star, 1}$
}

Tissue-type plasminogen activator (tPA) is a pleiotropic serine protease of the central nervous system (CNS) with reported neurotrophic and neurotoxic functions. Produced and released under its single chain form (sc), the sc-tPA can be cleaved by plasmin or kallikrein in a two chain form, tc-tPA. Although both sc-tPA and tc-tPA display a similar fibrinolytic activity, we postulated here that these two conformations of tPA (sc-tPA and tc-tPA) could differentially control the effects of tPA on neuronal survival. Using primary cultures of mouse cortical neurons, our present study reveals that Sc-tPA is the only one capable to promote $\mathrm{N}$ methyl-D-aspartate receptor (NMDAR)-induced calcium influx and subsequent excitotoxicity. In contrast, both sc-tPA and tc-tPA are capable to activate epidermal growth factor receptors (EGFRs), a mechanism mediating the antiapoptotic effects of tPA. Interestingly, we revealed a tPA dependent crosstalk between EGFR and NMDAR in which a TPA-dependent activation of EGFRs leads to downregulation of NMDAR signaling and to subsequent neurotrophic effects.

Cell Death and Disease (2015) 6, e1924; doi:10.1038/cddis.2015.296; published online 15 October 2015

Tissue-type plasminogen activator (tPA) is secreted by endothelial cells and promotes fibrinolysis via the conversion of fibrin-bound plasminogen into plasmin. ${ }^{1}$ Neurons and some glial cells also secrete tPA. ${ }^{2-5}$ tPA is secreted as a single-chain form (sc-tPA), which can be processed into a two-chain form (tc-tPA) by plasmin or kallikreins. ${ }^{6,7}$ Interestingly, sc-tPA is proteolytically active even without proteolytic processing. In addition to its vascular functions, tPA displays critical roles in the brain parenchyma with roles in cell migration, neuronal plasticity and survival, ${ }^{8-14}$ acting either as an enzyme or as a cytokine-like molecule. Among its actions, tPA is well described to promote neurotoxicity, likely through promotion of $\mathrm{N}$-methyl-D-aspartate receptor (NMDAR) activity. ${ }^{15-17}$ Recently, we reported that only sc-tPA can promote NMDAR signaling and neurotoxicity. ${ }^{18}$ Interestingly, data from wild-type mice, ${ }^{19}$ transgenic mice overexpressing tPA in neurons ${ }^{20}$ or in vitro ${ }^{21}$ also report neuroprotective effects of tPA. ${ }^{9,10}$ The proposed mechanisms involved a tPA-dependent and nonproteolytic activation of either epidermal growth factor receptors (EGFRs) ${ }^{22}$ on oligodendrocytes or NMDARs. ${ }^{20}$

Here we explored a link between tPA conformations (sc-tPA and tc-tPA), EGFR- and NMDAR-dependent signaling pathways. Our findings identify Sc-tPA as a selective positive modulator of NMDAR signaling in neurons when present at high concentrations and both Sc-tPA and tc-tPA as positive modulators of EGFR signaling, this even at low concentrations. We also reveal a crosstalk between these two families of receptors, with the tPA-dependent activation of EGFRs reducing NMDAR signaling. By these mechanisms, sc-tPA and tc-tPA control neuronal death and survival.

\section{Results}

sc-tPA promotes and tc-tPA inhibits NMDAR signaling. We first investigated NMDAR-induced calcium influx in the presence of either Sc-tPA or tc-tPA on primary cultures of cortical neurons (Figure 1). tc-tPA was produced from human recombinant purified sc-tPA (Actilyse, see Materials and Methods, Figure 1a). sc-tPA and tc-tPA were characterized and used at equimolarity, as previously described. ${ }^{18}$ When added on neurons, sc-tPA is rapidly converted into tc-tPA $(1 \mathrm{~h})$, an effect increased by plasmin $(5 \mathrm{nM})$. Moreover, the conversion of Sc-tPA into tc-tPA is blocked by aprotinin $(1 \mu \mathrm{M})$, an inhibitor of plasmin (Figure 1b). sc-tPA (300 nM) promoted NMDA-induced neuronal calcium influx ( $80 \%$ of cells potentiated; $27 \%$ of potentiaton; $\left.{ }^{*} P<0.0001\right)$. On the contrary, tc-tPA $(300 \mathrm{nM})$ led to a significant decrease in NMDAinduced calcium influx $(51 \%$ of cells inhibited; $11 \%$ of inhibition for tc-tPA $300 \mathrm{nM}$; ${ }^{\star} P<0.0001$; Figures $1 \mathrm{c}$ and d).

Both sc-tPA and tc-tPA can promote EGFR signaling. Immunoblottings for phosphorylated EGFRs (phosphotyrosines 1173 and 992) revealed that, similar to EGF $(50 \mathrm{ng} / \mathrm{ml})$, both sc-tPA and tc-tPA $(300 \mathrm{nM})$ can activate neuronal EGFRs (Y1173: Figures $2 \mathrm{~A}-\mathrm{C} ;+82 \%,+47 \%,+34 \%$ for EGF, sc-tPA and tc-tPA versus control, respectively;

\footnotetext{
${ }^{1}$ Inserm, Inserm U919, Serine Proteases and Pathophysiology of the Neurovascular Unit, University Caen Basse-Normandie, GIP Cyceron, Caen, France *Corresponding author: D Vivien, Inserm U919, GIP Cyceron, Bvd Becquerel, BP522914074, Caen 14074, France. Tel: +33 231470166 ; Fax: +33 2314702 22; E-mail: vivien@cyceron.fr

${ }^{2}$ These authors contributed equally to this work.

Abbreviations: DIV, days in vitro; EGF, epidermal growth factor; GluN1, NMDA receptor subunit 1; LRP, low-density lipoprotein receptor-related protein; NMDA, N-methylD-aspartate; Sc-tPA, single-chain tissue-type plasminogen activator; tc-tPA, two-chain tissue-type plasminogen activator.

Received 08.4.15; revised 27.7.15; accepted 17.8.15; Edited by A Yaron
} 
a

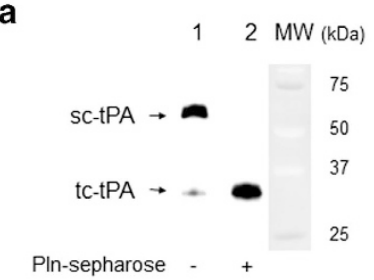

C

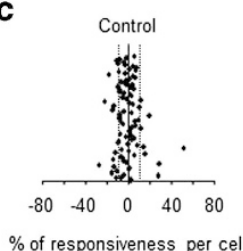

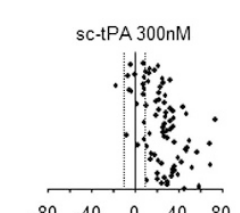

$\begin{array}{lllll}-80 & -40 & 0 & 40 & 80\end{array}$

$\%$ of responsiveness per cell
(post-incubation / pre-incubation)

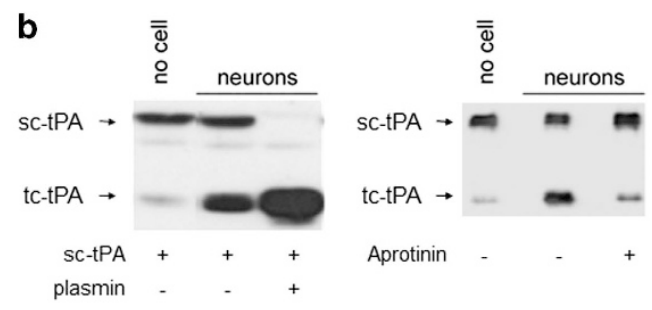

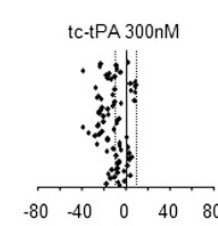

$\begin{array}{lllll}-80 & -40 & 0 & 40 & 80\end{array}$

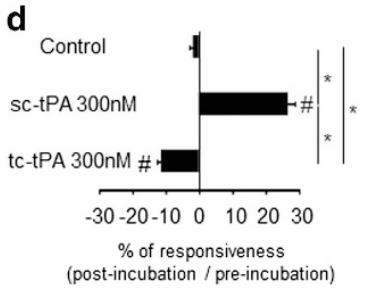

Figure 1 sc-tPA and tc-tPA differentially influence NMDAR signaling. (a) Sodium dodecyl sulfate-polyacrylamide gel electrophoresis (SDS-PAGE) followed by immunoblotting of sc-tPA and tc-tPA prepared as described in the Materials and Methods section (100 ng per lane). (b) SDS-PAGE followed by immunoblotting of sc-tPA and tc-tPA. Sc-tPA was added on cultured cortical neurons 13 DIV for $1 \mathrm{~h}$ either alone or in the presence of plasmin or aprotinin. (c) Calcium video imaging performed on primary cultures of cortical neurons (12 DIV). After control NMDA stimulations $(2 \times 25 \mu \mathrm{M}, 30 \mathrm{~s})$ used as baseline, neurons were incubated for 45 min in the presence of buffer (control, $n=90 \mathrm{cells}$ ), sc-tPA or tc-tPA at $300 \mathrm{nM}$ (sc-tPA, $n=85$ cells; tc-tPA, $n=78$ cells) prior to a second set of NMDA stimulations $(2 \times 25 \mu \mathrm{M}, 30 \mathrm{~s})$. Percentages of potentiation or inhibition after incubation are calculated for each cell. (d) Percentage of potentiation or inhibition after incubation for each group (mean \pm S.E.M.; ${ }^{*} P<0.0001$ Kruskal-Wallis test followed by Mann-Whitney test; ${ }^{\#} P<0.0001$ Wilcoxon test comparison of preincubation and postincubation responses)

${ }^{*} P<0.05$ and $Y 992$ : Figures $2 \mathrm{D}-\mathrm{F} ;+56 \%,+74 \%,+52 \%$ for EGF $50 \mathrm{ng} / \mathrm{ml}$, sc-tPA and tc-tPA versus control, respectively; ${ }^{*} P<0.05$ ). Proximity ligation assays (Figures $2 \mathrm{G}$ and $\mathrm{H}$ ) and immunoprecipitation-immunoblotting assays (Figure 2l) revealed that NMDARs (NMDA receptor subunit 1 (GluN1) subunit) form complexes with EGFRs. Interestingly, EGF led to a reduction of NMDA-induced neuronal calcium influx ( $57 \%$ of cells inhibited; $11 \%$ of inhibition for EGF; ${ }^{*} P<0.0001$ when compared with controls; Figures $2 \mathrm{~J}$ and $\mathrm{K}$ ).

tPA-dependent crosstalk between NMDARs and EGFRs. NMDAR-induced calcium influx was studied in the presence of sc-tPA and tc-tPA $(300 \mathrm{nM})$ alone or in the presence of either an inhibitor of the transphosphorylation of EGFRs (AG1478) or a GluN1 antibody previously characterized to prevent TPA-induced potentiation of NMDAR signaling ${ }^{23}$ (Figures 3 and 4). The potentiating effect of sc-tPA on NMDA-induced calcium influx $(85 \%$ of cells potentiated; $25 \%$ of potentiation for sc-tPA $300 \mathrm{nM}$; ${ }^{*} P<0.0001$ when compared with controls) was completely prevented by GluN1 antibody (NS when compared with controls; ${ }^{*} P<0.0001$ when compared with sc-tPA alone; Figure $3 a-c)$. In contrast, the presence of AG1478 failed to influence tPA-promoted NMDAR signaling $(77 \%$ of cells potentiated; $19 \%$ of potentiation for sc-tPA $300 \mathrm{nM}+\mathrm{AG} 1478$; ${ }^{*} P<0.0001$ when compared with controls and NS: not significant when compared with sc-tPA alone, Figure 3d-f). In parallel, blockage of the trans-activation of EGFRs by AG1478 (Figures $4 \mathrm{a}-\mathrm{C}$ ) prevented tc-tPA-dependent inhibition of NMDAR signaling $\left({ }^{\star} P<0.0001\right.$ considering the percentage of responsiveness for tc-tPA $300 \mathrm{nM}$ alone versus tc-tPA $300 \mathrm{nM}+\mathrm{AG} 1478 ; 46 \%$ versus $15 \%$ of cells inhibited in tc-tPA $300 \mathrm{nM}$ and tc-tPA $300 \mathrm{nM}+\mathrm{AG} 1478$, respectively). tc-tPA-induced inhibition of NMDAR-mediated calcium influx was not modulated by the co-application of the GluN1 antibody (54\% of cells inhibited; $10 \%$ of inhibition for tc-tPA $300 \mathrm{nM}$ alone compared with $59 \%$ of cells inhibited and $11 \%$ of inhibition for tc-tPA $300 \mathrm{nM}+\mathrm{GluN1}$ antibody; ${ }^{*} P<0.0001$; Figures $4 \mathrm{~d}-\mathrm{f}$ ). Parallel experiments performed using another inhibitor of the activation of EGFR, Gefitinib $(5 \mu \mathrm{M})$, provided the same results as observed in the presence of AG1478 (Supplementary Figure 2). Altogether, these data reveal a crosstalk between NMDAR signaling and EGFRs in which tPA-mediated EGFR activation leads to an inhibition of NMDAR signaling.

In a paradigm of NMDA-mediated excitotoxicity, only the sc-tPA promotes neuronal death. NMDA-mediated excitotoxicity was tested on primary cultures of cortical neurons subjected to NMDA exposure in the presence of Sc-tPA or tc-tPA $(300 \mathrm{nM})$. As expected, NMDA-induced excitotoxicity was potentiated only in the presence of sc-tPA $(+47 \%$; ${ }^{*} P<0.05$; Figure 5a). Co-application of tPA stop, an inhibitor of the proteolytic activity of tPA ${ }^{24}$ or the GluN1 antibody (see Figure 3 ) prevented the pro-excitotoxic effect of Sc-tPA $\left({ }^{\star} P<0.05\right.$; Figures $5 b$ and $\left.c\right)$.

In a paradigm of serum deprivation (SD)-induced apoptosis, both Sc-tPA and tc-tPA are neuroprotective, an effect dependent on EGFR signaling. In a paradigm of apoptotic neuronal death induced by deprivation of trophic factors in cortical neurons, both sc-tPA and tc-tPA displayed antiapoptotic properties. Aprotinin failed to prevent the antiapoptotic effect of sc-tPA, suggesting that the antiapoptotic effect of sc-tPA is not due to its previous conversion into tc-tPA $\left({ }^{*} P<0.05\right.$; Figure 6a). Blockage of the ability of tPA to promote NMDAR-induced calcium influx with the GluN1 antibody did not prevent the antiapoptotic effects of both 

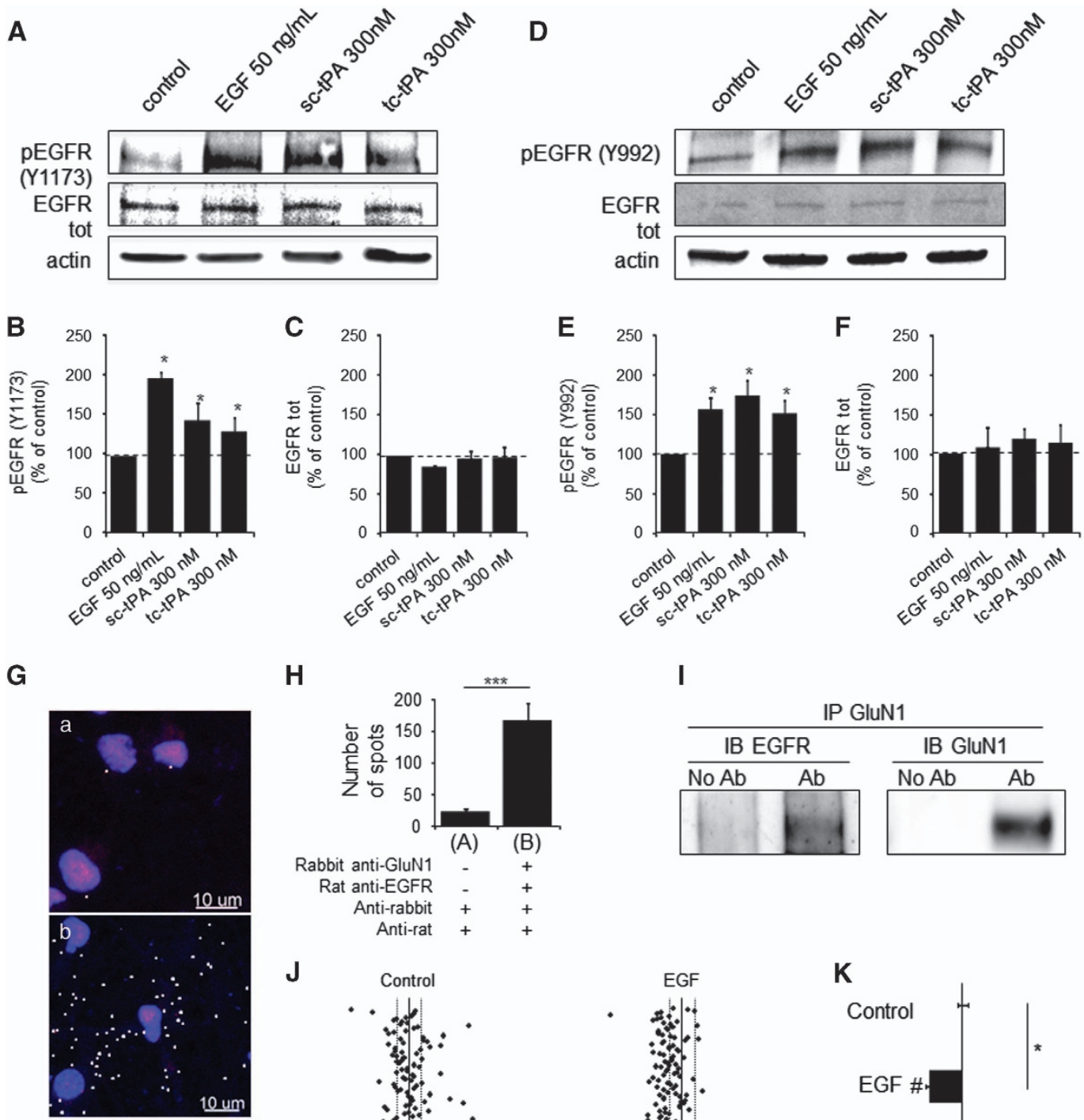

H

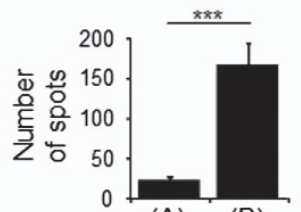

(A)
I

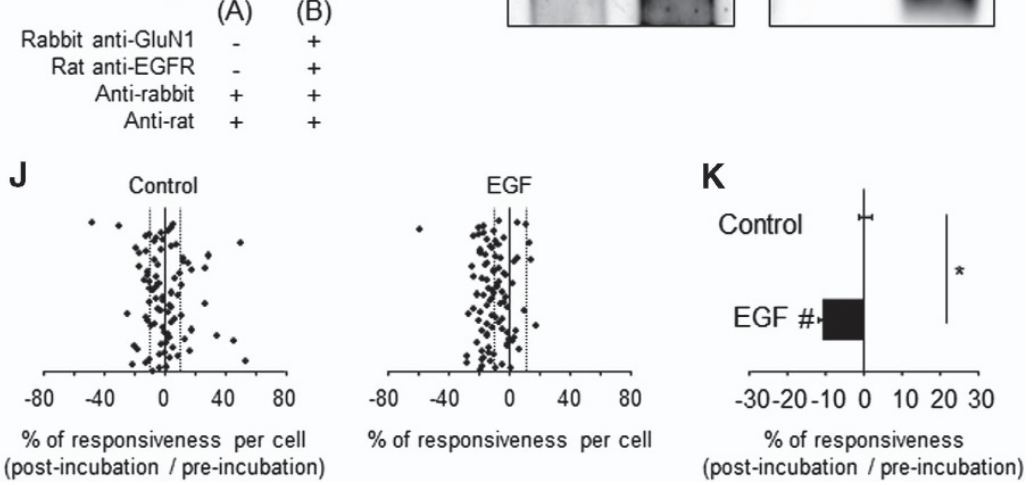

Figure 2 Both sc-tPA and tc-tPA at $300 \mathrm{nM}$ can modulate EGFR signaling. (a) Representative immunoblots for phospho-EGFR (A-C: tyrosine 1173; D-F: tyrosine 992) and total EGFR on neurons (12-13 DIV) after treatments with EGF (50 ng/ml), sc-tPA or tc-tPA ( $300 \mathrm{nM})$ during $15 \mathrm{~min}$. (B, C, E and F) Quantifications of phosphorylated EGFRs and total EGFRs were compared with control (mean \pm S.E.M.; $N=3$ or 4 experiments; ${ }^{*} P<0.05$ ). (g) Confocal images of endogenous NMDAR-EGFR complexes in cortical neurons. (H) Quantification of NMDAR-EGFR complexes detected by Proximity Ligation Assay (PLA). ${ }^{* \star *} P$-value $<0.001$; Mann-Whitney $U$-test, $N=3$. (I) Crossimmunoprecipitation assays demonstrating that EGFRs and NMDARs form stable complexes in cultured neurons. (J) After two NMDA stimulations used as baseline, neurons were incubated for $45 \mathrm{~min}$ with buffer (control, $n=85 \mathrm{cells}$ ) or EGF $50 \mathrm{ng} / \mathrm{ml}$ (EGF, $n=93 \mathrm{cells}$ ) prior to a second set of NMDA stimulations. Percentages of potentiation or inhibition after incubation are calculated for each cell. (K) Percentage of potentiation or inhibition after incubation for each group (mean \pm S.E.M.; ${ }^{*} P<0.0001 \mathrm{Kruskal}-$ Wallis test followed by Mann-Whitney U-test; ${ }^{\#} P<0.0001$ Wilcoxon test comparison of preincubation and postincubation responses)

sc-tPA and tc-tPA ( ${ }^{\star} P<0.05$, Figure $\left.6 \mathrm{~b}\right)$. However, blockage of the tPA-dependent transphosphorylation of EGFRs (AG1478) prevented the antiapoptotic activities of both sc-tPA and tc-tPA ( ${ }^{\star} P<0.05$, Figure $\left.6 c\right)$.

Low concentrations of sc-tPA and tc-tPA are neurotrophic, an effect mediated by a crosstalk between EGFRs and NMDARs. As it was previously reported that low concentrations of tPA may have protective effects through activation of NMDARs, ${ }^{20}$ we tested lower concentrations of
sc-tPA and tc-tPA (10 nM; Figure 7) in our different paradigms. Immunoblotting for phosphorylated EGFRs revealed a sc-tPA- and tc-tPA-dependent (10 nM) activation of the EGFRs $(+82$ and $+154 \%$ of activation for sc-tPA and tc-tPA at $10 \mathrm{nM}$, respectively; ${ }^{\star} P<0.05$; Figures $7 \mathrm{a}-\mathrm{c}$ ). Parallel experiments using calcium video microscopy revealed that Sc-tPA and tc-tPA $(10 \mathrm{nM})$ led to an inhibition of NMDAR signaling (78 and $63 \%$ of cells inhibited; 17 and $14 \%$ of inhibition for sc-tPA and tc-tPA $(10 \mathrm{nM})$, respectively; $\left.{ }^{\star} P<0.0001\right)$. This inhibitory effect was blocked by the 


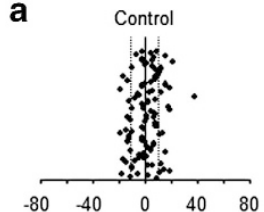

$\%$ of responsiveness per cell (post-incubation / pre-incubation)

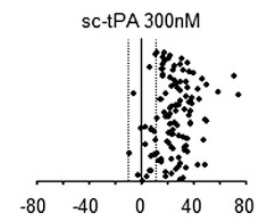

$\%$ of responsiveness per cell

b

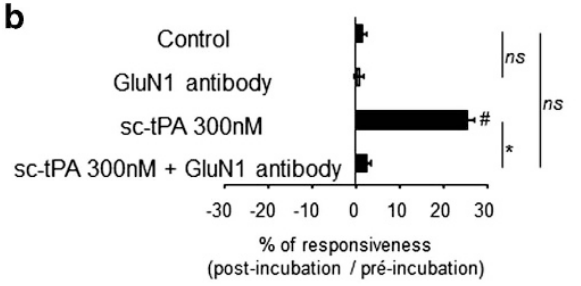

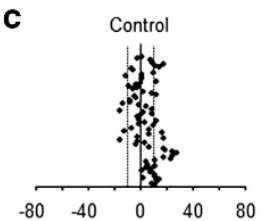

$\%$ of responsiveness per cell (post-incubation / pre-incubation)

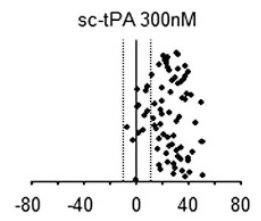

$\%$ of responsiveness per cell

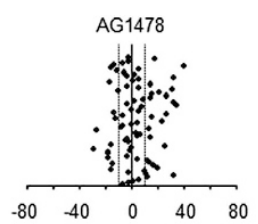

$\%$ of responsiveness per cell

sc-tPA 300nM + AG1478

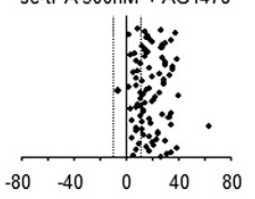

$\%$ of responsiveness per cell

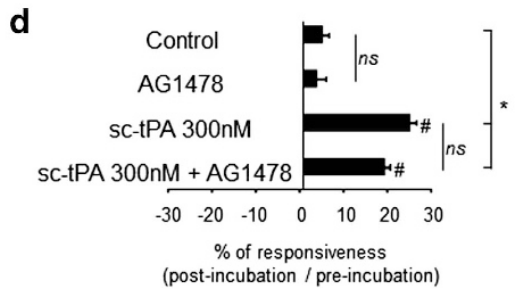

Figure 3 sc-tPA-promoted neuronal calcium influx is dependent on its interaction with NMDARs. Calcium video imaging performed on cortical neurons. (a) After two control NMDA stimulations used as baseline, neurons were incubated for $45 \mathrm{~min}$ in the presence of either buffer (control, $n=104 \mathrm{cellls}$ ), sc-tPA at $300 \mathrm{nM}$ (sc-tPA $n=111$ cells) or GluN1 antibody at $10 \mu \mathrm{g} / \mathrm{ml}$ ( $n=99$ cells) alone or in combination (sc-tPA+GluN1 antibody; $n=109$ cells) prior to a second set of NMDA stimulations. Percentages of potentiation or inhibition after treatment are calculated for each cell. (b) Percentages of potentiation or inhibition after treatment are calculated for each cell and reported as percentages of responsiveness for each group. (c) In the same protocol, neurons were incubated for $45 \mathrm{~min}$ in the presence of buffer (control, $n=75 \mathrm{cells}$ ), sc-tPA at $300 \mathrm{nM}$ (sc-tPA, $n=77 \mathrm{cells}$ ) or AG1478 at $5 \mu \mathrm{M}$ (AG1478, $n=77$ cells) alone or in combination (sc-tPA+AG1478, $n=90$ cells) prior to a second set of NMDA stimulations ( $2 \times 25 \mu \mathrm{M}, 30 \mathrm{~s}$ ). (d) Percentages of responsiveness for each group (mean \pm S.E.M.; ${ }^{*} P<0.0001$ Kruskal-Wallis test followed by Mann-Whitney test; ${ }^{\#} P<0.0001$ Wilcoxon test comparison of preincubation and postincubation responses). NS: not significant

co-application of AG1478 ( 3 and $11 \%$ of cells inhibited; 7 and $8 \%$ of potentiation for Sc-tPA and tc-tPA at $10 \mathrm{nM}+\mathrm{AG} 1478$, respectively; ${ }^{*} P<0.0001$; Figures $7 \mathrm{~d}-\mathrm{f}$ ). As an additional control, tc-tPA at $1 \mathrm{nM}$ did not influence NMDA-induced calcium influx (Supplementary Figure 1).

Although sc-tPA at $300 \mathrm{nM}$ promoted NMDAR-mediated excitotoxicity, $10 \mathrm{nM}$ of either sc-tPA or tc-tPA protected neurons in the same paradigm of NMDAR-mediated excitotoxicity $(-65 \%$ of excitotoxic death for sc-tPA, $-60 \%$ for tc-tPA $10 \mathrm{nM}$; ${ }^{*} P<0.05$, Figure $7 \mathrm{~g}$ ). Interestingly, blockage of the transphorylation of EGFRs (AG1478; Figure $7 \mathrm{~g}$ ) reversed these neuroprotective actions of sc-tPA and tc-tPA. As expected, both sc-tPA and tc-tPA displayed antiapoptotic properties even at low concentrations $(-59 \%$ of apoptotic death at $300 \mathrm{nM}$ and $-35 \%$ at $10 \mathrm{nM}$; ${ }^{*} P<0.05$; Figure $7 \mathrm{~h}$ ).

Altogether, these data demonstrate that although direct activation of NMDARs by sc-tPA at high concentrations led to a pro-excitotoxic effect dependent of its proteolytic activity, lower concentrations of both Sc-tPA and tc-tPA are antiexcitotoxic by a mechanism involving an EGFR-dependent downregulation of NMDAR signaling independently of their proteolytic activity. Our data also evidence that both Sc-tPA and tc-tPA display antiapoptotic functions through a mechanism involving a direct activation of EGFRs and this independently of NMDARs (Figure 8).

\section{Discussion}

We propose here a new scheme of the mechanisms through which IPA controls neuronal survival. We show that both conformations (sc-tPA and tc-tPA) have a neurotrophic effect by the activation of EGFRs. EGFRs can complex to NMDARs at the neuronal surface, orchestrating an original tPA-dependent crosstalk between both receptors, leading to a downregulation of NMDAR signaling and subsequent neurotrophic effects. However, when present at high concentration $(300 \mathrm{nM})$, the Sc-tPA promotes NMDAR signaling leading to an increased neuronal death, hiding the neurotrophic effects of lower concentrations of tPA.

tPA-driven control of neuronal fate could also depend on the different subtypes of NMDAR subunits involved, as well as on the location of the receptors (synaptic versus extrasynaptic). Specifically, exogenous tPA can not only promote neurotoxicity on cortical neurons by activating extrasynaptic GluN2Dcontaining NMDARs ${ }^{25}$ but can also activate synaptic GluN2A-containing NMDARs, leading to a neuroprotective effect. ${ }^{19}$ Alternatively, the neurotoxic versus neuroprotective effects of tPA may reflect different effects of endogenous versus exogenous IPA or of chronic versus acute treatments. Thus, as previously suggested, ${ }^{20}$ our present data show that tPA may have opposite effects depending on its concentration, with the low concentrations that are protective and the higher concentrations of Sc-tPA that are deleterious. In addition, we 
a

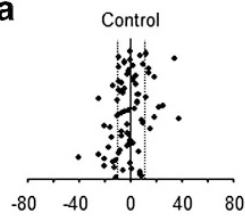

$\%$ of responsiveness per cell (post-incubation / pre-incubation)

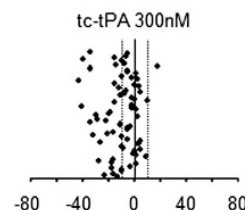

$\%$ of responsiveness per cell

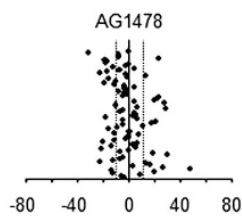

$\%$ of responsiveness per cell
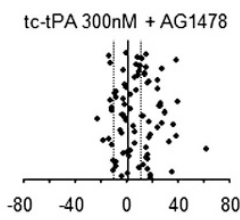

$\%$ of responsiveness per cell
C

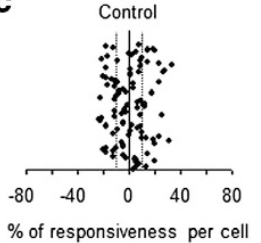

$\%$ of responsiveness per cell

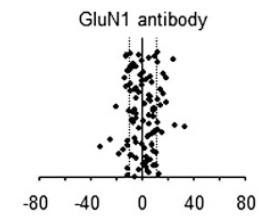

$\%$ of responsiveness per cell

$$
\text { tc-tPA 300nM + GluN1 antibody }
$$

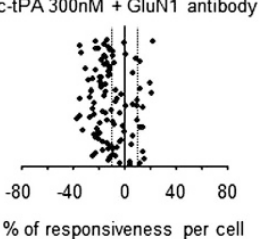

$\%$ of responsiveness per cell b

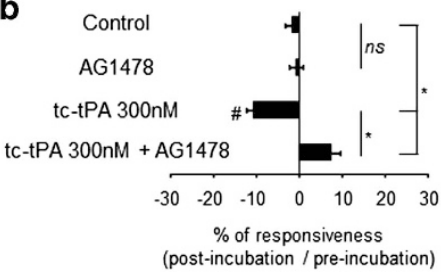

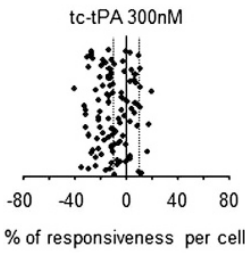

d

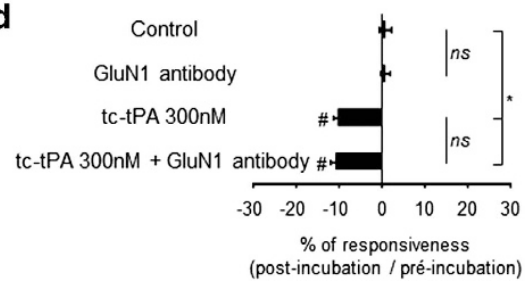

Figure 4 AG1478 reverses the inhibitory effect of tc-tPA on NMDAR signaling independently of its interaction with NMDARs. (a) After two NMDA stimulations used as baseline, neurons were incubated for $45 \mathrm{~min}$ in the presence of buffer (control, $n=74$ cells), AG1478 at $5 \mu \mathrm{M}$ (AG1478, $n=84$ cells) or tc-tPA at $300 \mathrm{nM}$ alone or in combination (tc-tPA, $n=70$ cells; tc-tPA+AG1478, $n=81$ cells) prior to a second set of NMDA stimulations. Percentages of potentiation or inhibition after incubation are calculated for each cell. (b) Percentages of potentiation or inhibition after incubation are calculated for each individual cell and reported as percentages of responsiveness for each group. (c) In the same protocol, neurons were incubated for $45 \mathrm{~min}$ in the presence of buffer (control, $n=99 \mathrm{cells}$ ), GluN1 antibody at $10 \mu \mathrm{g} / \mathrm{ml}$ (GluN1 antibody, $n=99 \mathrm{cells}$ ) or tc-tPA at $300 \mathrm{nM}$ either alone or in combination tc-tPA $300 \mathrm{nM} n=103$ cells, tc-tPA+GluN1 antibody $n=106$ cells) prior to a second set of NMDA stimulations $(2 \times 25 \mu \mathrm{M}, 30 \mathrm{~s})$. (d) Percentages of potentiation or inhibition after incubation are calculated for each individual cell and reported as percentages of responsiveness for each group (mean \pm S.E.M.; ${ }^{*} P<0.0001$, Kruskal-Wallis test followed by Mann-Whitney test; ${ }^{\#} P<0.0001$ Wilcoxon test of comparison of preincubation and postincubation responses). NS: not significant
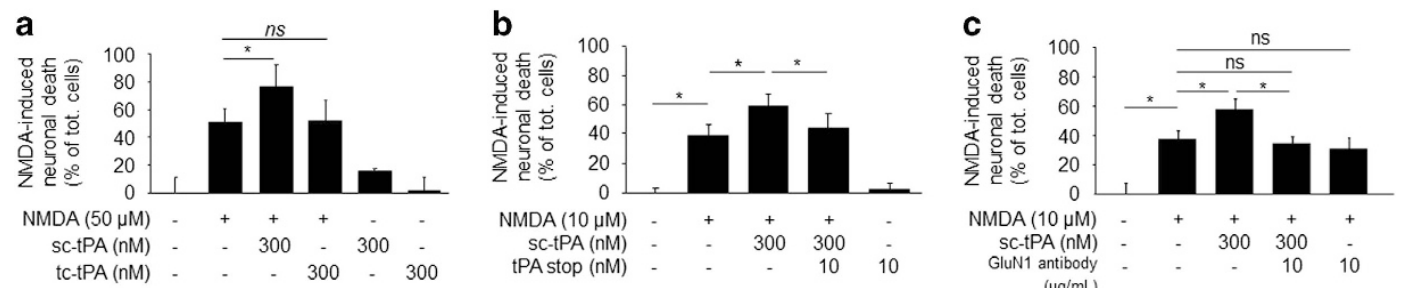

Figure 5 Only high concentrations of active sc-tPA promote excitotoxicity. (a) Cortical neurons (12-13 DIV) were exposed for $1 \mathrm{~h}$ to NMDA ( $50 \mu \mathrm{M})$ in the presence of sc-tPA or tc-tPA. Neuronal death was quantified $24 \mathrm{~h}$ later. (b and c) Same experiments as in panel (a) were performed with neurons exposed for $24 \mathrm{~h}$ to NMDA (10 $\mu \mathrm{M})$ in the presence of sc-tPA alone or in combination with tPA stop ( $10 \mathrm{nM}$, b) or GluN1 antibody $(10 \mu \mathrm{g} / \mathrm{ml}, \mathbf{c})$. (mean \pm S.E.M.; $n=3$ experiments; 4 wells per condition; ${ }^{*} P<0.05$, NS: not significant, Kruskal-Wallis test followed by Mann-Whitney test)

a

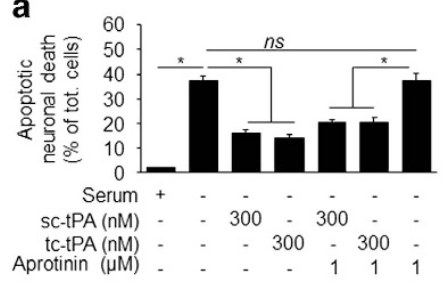

b

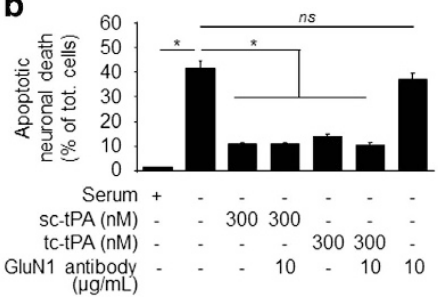

C

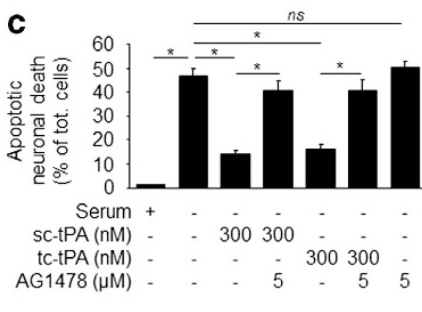

Figure 6 Both sc-tPA and tc-PAA rescue neurons from serum deprivation-induced apoptosis. (a) Neuronal death measured after a 24-h exposure to serum deprivation (SD) alone or in the presence of Sc-tPA or tc-tPA at $300 \mathrm{nM}$ alone or plus aprotinin $\left(1 \mu \mathrm{M}\right.$; mean \pm S.E.M.; $n=3$ experiments; $\left.{ }^{*} P<0.05\right)$. (b and $\left.\mathbf{c}\right)$ Neuronal death measured after a 24-h exposure to SD alone or in the presence of sc-tPA or tc-tPA plus GluN1 antibody $(10 \mu \mathrm{g} / \mathrm{ml} ; \mathbf{b})$ or AG1478 $(5 \mu \mathrm{M} ; \mathrm{c})(\mathrm{mean} \pm \mathrm{S}$.E.M.; $n=3$ experiments; 4 wells per condition; ${ }^{*} P<0.05$, NS: not significant, Kruskal-Wallis test followed by Mann-Whitney test) 

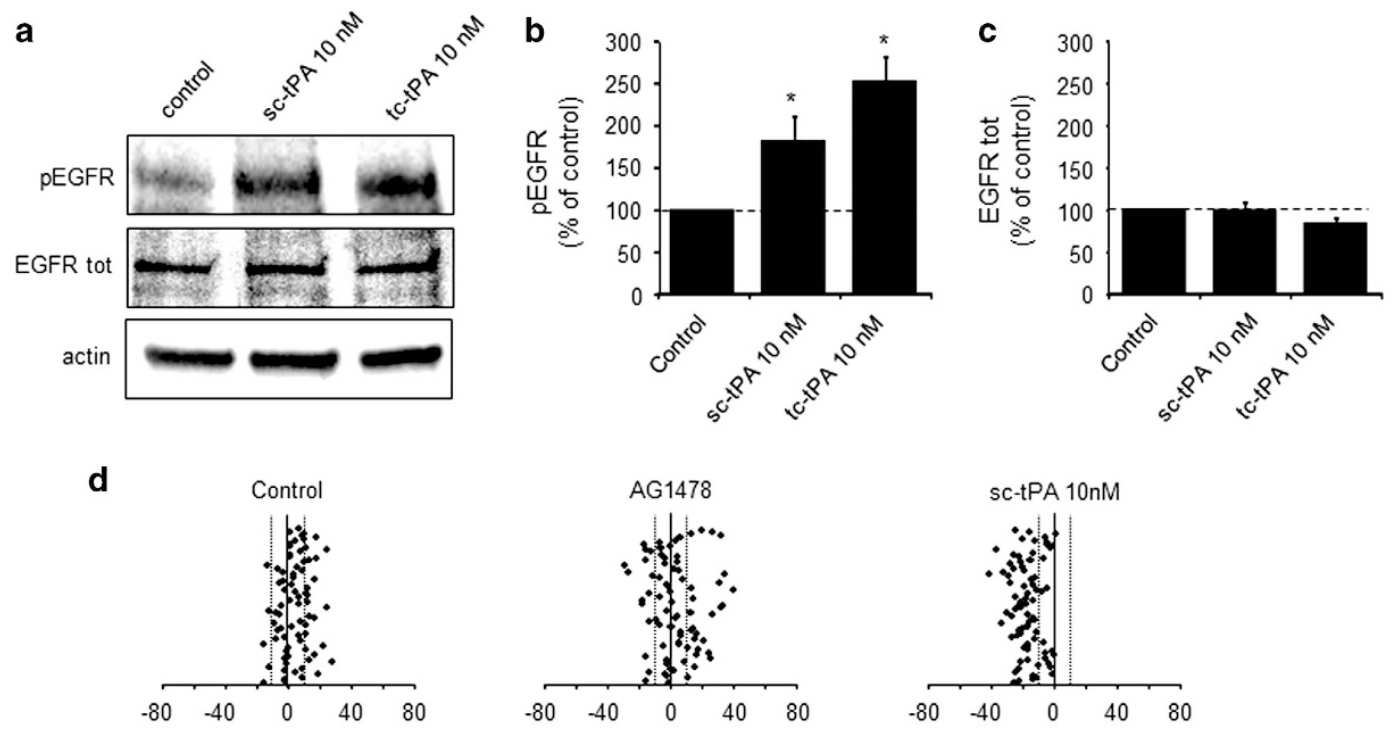

$\%$ of responsiveness per cell (post-incubation / pre-incubation)

$\%$ of responsiveness per cell

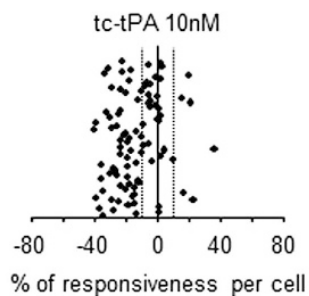

$\%$ of responsiveness per cell

tc-tPA $10 n M+A G 1478$

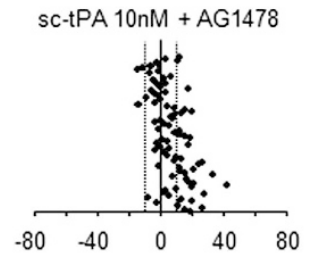

$\%$ of responsiveness per cell

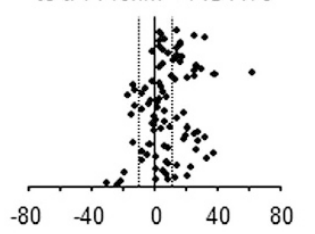

$\%$ of responsiveness per cell

e
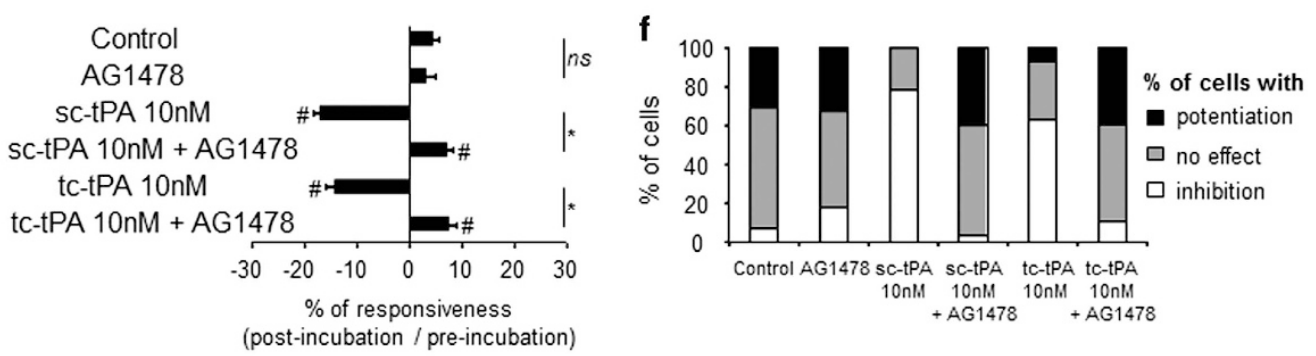

g

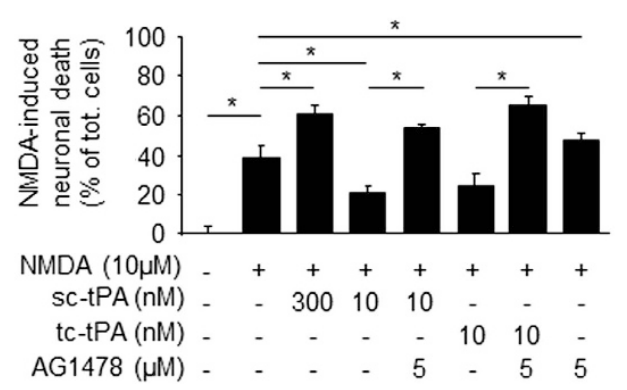

h

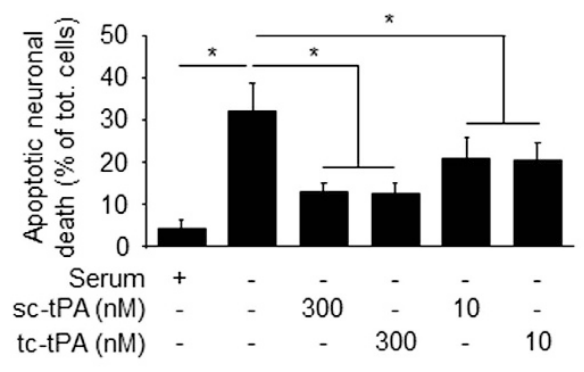

Figure 7 Both Sc-tPA and tc-tPA at $10 \mathrm{nM}$ promote EGFR signaling and are neuroprotective. (a) Representative immunoblots for phospho-EGFR (tyrosine 1173) and total EGFR on neurons after treatments with sc-tPA and tc-tPA $(10 \mathrm{nM})$ during $15 \mathrm{~min}$. (b and $\mathbf{c})$ Quantification of phosphorylated EGFRs and total EGFRs compared with the control condition ( $n=3$ experiments; ${ }^{*} P<0.05$ ). (d) After two NMDA stimulations used as baseline, neurons were incubated for 45 min in the presence of buffer (control, $n=75$ cells), AG1478 at $5 \mu \mathrm{M}(n=77$ cells), sc-tPA (10 nM) alone or in combination with AG1478 (sc-tPA, $n=78$ cells; sc-tPA+AG1478, $n=83$ cells) or tc-tPA (10 nM) alone or in combination with AG1478 (tc-tPA, $n=92$ cells; tc-tPA+AG1478, $n=92$ cells) prior to a second set of NMDA stimulations. Percentages of potentiation or inhibition after incubation are calculated for each cell. (e) Percentages of responsiveness for each group (mean \pm S.E.M.; ${ }^{*} P<0.0001$ Kruskal-Wallis test followed by Mann-Whitney test; ${ }^{\#} P<0.0001$ Wilcoxon test comparison of preincubation and postincubation responses). (f) Percentages of cells either potentiated, inhbited or without effect for each group. (g) Cortical neurons were subjected to 24-h exposure to NMDA (10 $\mu \mathrm{M})$ in the presence of either Sc-tPA or tc-tPA (10 nM) alone or in combination with AG1478 (5 $\mu \mathrm{M} ; n=3$ experiments; 4 wells per condition; ${ }^{\star} P<0.05$, NS: not significant; Kruskal-Wallis test followed by Mann-Whitney test). (h) Neuronal death measured after a 24-h exposure to either serum deprivation (SD) alone or in the presence of either sc-tPA or tc-tPA at 300 or $10 \mathrm{nM}\left(n=3\right.$ experiments; 4 wells per condition experiments; ${ }^{*} P<0.05$, NS: not significant; KruskalWallis test followed by Mann-Whitney test, mean \pm S.E.M.) 


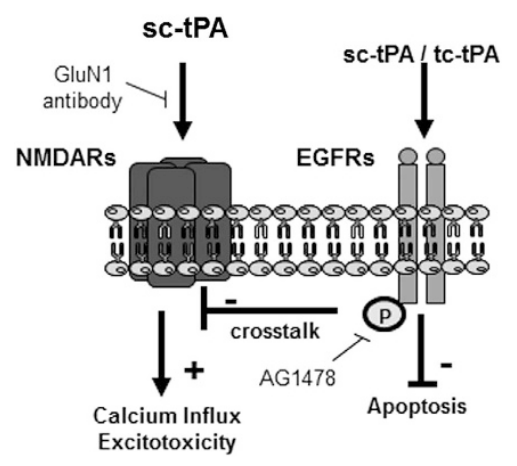

Figure 8 tPA-dependent crosstalk between EGFRs and NMDARs. Sc-tPAinduced potentiation of NMDAR signaling and subsequent neurotoxicity, inhibited by GluN1 antibody. tPA also promotes EGFR signaling and subsequent antiapoptotic effects, independently of its conformation (sc-tPA and tc-tPA), an effect occurring even at low concentrations (down to $10 \mathrm{nM}$ ) and inhibited by AG-1478, an inhibitor of EGFR transphosphorylation. tPA-dependent activation of EGFRs leads to downregulation of NMDAR signaling and subsequent neurotrophic effects

propose here that tPA may differentially influence neuronal fate and signaling pathways as a function of its conformation with sc-tPA and tc-tPA.

Endogenous tPA is produced and released under its single chain-form (sc-tPA) ${ }^{26}$ and is $90 \%$ present under its singlechain form in Alteplase when used for thrombolysis (see Figure $1 b$, lane no cell). When released, it can be rapidly converted into its two-chain form (tc-tPA) by plasmin ${ }^{26,27}$ present at the cell surface or in solution. Thus endogenous plasmin may directly influence the sc-tPA/tc-tPA ratio. It is now well admitted that the tPA-mediated potentiation of NMDAR signaling is dependent on its proteolytic activity. ${ }^{15,17}$ However, both plasmin-dependent and plasmin-independent mechanisms have been reported. ${ }^{15,17,28}$ Here we demonstrate that potentiation of NMDAR signaling and subsequent neurotoxicity is a phenomenon restricted to Sc-tPA and dependent of proteolytic activity. Whether tPA would require low-density lipoprotein receptor-related protein (LRP) or not in order to enhance NMDAR signals could depend on the type of neurons, their state of maturation or the presence of astrocytes in cultures. ${ }^{17,29}$

Several in vitro studies reported antiapoptotic effects of tPA on neurons ${ }^{9,10}$ and oligodendrocytes. ${ }^{22}$ In agreement with our data, despite the heterogeneity of the toxic paradigms used, they all show that this trophic function of tPA occurs independently of its proteolytic activity. Two candidates have been proposed as the receptors mediating the antiapoptotic effects of tPA: Annexin II and EGFRs. ${ }^{10,22}$ For instance, tPA can bind EGFRs on oligodendrocytes through its EGF-like domain, induces phosphorylation of EGFRs and subsequent signaling pathways, leading to antiapoptotic effects. We evidenced here that both sc-tPA and tc-tPA are antiapoptotic in neurons through a mechanism involving a EGFRdependent pathway.

Accordingly, data from studies in transgenic mice overexpressing tPA in neurons (T4 mice) suggested that TPA can have neuroprotective effects. ${ }^{19,20}$ In one of these studies, the authors propose a mechanism that is dependent of the activation of NMDARs and independent on plasmin. It is interesting to note that, in our hands, the tPA-dependent activation of EGFRs led to a reduced NMDAR-mediated calcium influx. These data unmask a tPA-dependent crosstalk between NMDARs and EGFRs, with NMDAR and EGFR that can form complexes. Similarly, in PC12 cells (pheochromocytoma cells) tPA was reported to control a crosstalk between NMDARs and Trk receptors, a mechanism that involves LRP1 and that is differentially controlled by the dose of tPA. ${ }^{30}$

In conclusion, our present study provides information that help to understand how tPA can positively or negatively control neuronal fate.

\section{Materials and Methods}

Experimental procedures. Experiments were carried out in accordance with the European Communities Council Directive (86/609/EEC) and were approved by the local ethical committee.

Chemicals. NMDA, (+)5-methyl-10,11-dihydro-5H-dibenzo(a,d)cyclopentaen-5, 10-imine maleate (MK801) and N-(3-Chlorophenyl)-6,7-dimethoxy-4-quinazolinanine hydrochloride (AG1478) were purchased from Tocris (Bristol, United Kingdom). 2,7-Bis-(4-amidinobenzylidene)-cycloheptanone-1-dihydrochloride salt (tPA-stop) was purchased from American Diagnostica (Stamford, CT, USA). Trasylol (aprotinin) was a gift from Bayer HealthCare AG. Iressa (Gefitinib) was a purchased from Selleckchem (Houston, TX, USA). 6-Aminocaproic acid, ascorbic acid, bFGF, Dulbecco's modified Eagle's medium (DMEM), HEPES buffer solution, hydrocortisone, poly-D-lysine, cytosine $\beta$-D-arabinoside, Arginine, Tween-80, phosphoric acid and $0.4 \%$ Trypan Blue Solution, fetal bovine serum and horse serum were from Sigma-Aldrich (L'Isle d'Abeau, France). CNBr-activated Sepharose 4B was from GE Healthcare (Orsay, France). Plasmin was prepared as described. ${ }^{30}$ Laminin, lipid concentrate and penicillin-streptomycin were purchased from Invitrogen (Cergy Pontoise, France). Endothelial cell basal medium (EBM-2) was purchased from Lonza (Levallois, France).

Sources of tPA. A human recombinant tPA purified from Chinese Hamster Ovary cells (Actilyse, $>95 \%$ single-chain) was used as single chain IPA (sc-tPA). Two-chain IPA (tc-tPA) was prepared by overnight incubation of sc-tPA with plasmincoupled Sepharose $4 \mathrm{~B}$ at $37^{\circ} \mathrm{C}$, followed by a $2 \mathrm{~h}$ incubation with immobilized aprotinin to eliminate traces of free plasmin. Both sc-tPA and tc-tPA were dialyzed in a vehicle containing $0.5 \mathrm{M}$ ammonium bicarbonate for neurotoxocity studies or in the Actilyse buffer (arginine, phosphoric acid and Tween-80) for video calcium imaging. Finally, both Sc-tPA and tc-tPA were characterized as we previously described. ${ }^{18}$

Neuronal cell cultures. Culture of cortical neurons were prepared from fetal mice (E14-15) as previously described. ${ }^{25}$ Cytosine $\beta$-D-arabinoside $(10 \mu \mathrm{M})$ was added after 3 days in vitro (DIV) to inhibit glial proliferation. Various treatments were performed either after 7 DIV for apoptotic paradigms or 12-13 DIV for NMDAmediated neurotoxicity assays, calcium imaging experiments and immunoblottings.

tPA and EGFR immunoblottings. tPA immunoblottings were performed using a polyclonal goat antibody (Santa Cruz Biotechnology - sc5239; 1:1000, Heidelberg, Germany) followed by incubation with the appropriate peroxidaseconjugated secondary antibody. Phospho EGFR (Tyr 1173), total EGFR and actin immunoblottings were performed using a polyclonal rabbit antibody (Cell Signaling 4407; 1:1000, Leiden, The Netherlands), a polyclonal rabbit antibody (Cell Signaling - 4267; 1:1000) and a polyclonal rabbit antibody (Sigma Aldrich A2066.2 ml; 1:1000), respectively, followed by incubation with the appropriate peroxidase-conjugated secondary antibody. Immunoblots were revealed with an enhanced chemoluminescence ECL2 immunoblotting detection system (Fisher Scientific, IIlkirch, France) and imaged in an ImageQant LAS 4000 Device (GE Heathcare, Orsay, France).

Proximity ligation assay. The proximity ligation assay was performed using the Duolink In Situ Kit (Olink Bioscience, Uppsala, Sweden) according to the manufacturer's instructions with the following modifications: PLA probe incubation was of $2 \mathrm{~h}$; amplification step was extended to $2 \mathrm{~h}$. Blocking ( $1 \mathrm{~h}$ at room temperature) and primary antibody (overnight at $4{ }^{\circ} \mathrm{C}$ ) incubations were performed in a $4 \%$ bovine serum albumin (Sigma Aldrich) and $0.2 \%$ Triton X-100 solution. 
Rabbit anti-GluN1 (ab17345, Abcam, Cambridge, MA, USA) and rat anti-EGFR (ab231, Abcam) were diluted $(1: 2000$ and $1: 100$, respectively) in the blocking solution. The anti-rabbit $(+)$ PLA probe $(1: 5)$ along with an anti-rat $(-)$ probe $(1: 100)$ were diluted in the blocking solution. A Goat anti-rat (Jackson ImmunoResearch Inc., Suffok, UK) were used to make a probe anti-rat according to the manufacturer's instructions using the Duolink Probemaker (Olink Bioscience). Slides were mounted in a mounting medium containing DAPI (4',6-diamidino-2phenylindole) and $0.1 \%$ deparaphenylene-diamine diluted in phosphate-buffered saline and glycerin. The negative control represents the PLA without the primary antibodies.

Ten stack of picture $(0.40 \mu \mathrm{m}$ per section) were taken from 10 different areas of every well with a confocal microscope (Leica SP5, Leica, Nanterre, France). Punctuas were counted manually using a $z$ projection of the stack.

Crossed immunoprecipitation assays. Supernatants from TNT buffer (50 mM Tris-HCl, pH 7.4, $150 \mathrm{mM} \mathrm{NaCl}$ and $0.5 \%$ Triton X-100)-lysed cultured cortical neurons (12 DIV) (500 $\mu \mathrm{g}$ of total proteins) were incubated overnight at $4{ }^{\circ} \mathrm{C}$ with an antibody raised against the C-terminal end of the GluN1 subunit of NMDAR ( $2 \mu \mathrm{g}$, Santa Cruz Biotechnology - sc1467) and then coupled to protein G-sepharose beads as described by the manufacturer (GE Healthcare) for immunoprecipation procedures. Then immunoprecipitated proteins were separated by $7.5 \%$ SDS-PAGE, and immunoblots were revealed with either an antibody raised against total EGFRs (Cell Signaling - 4267; 1:1000) or an antibody targeting the C-terminal end of the GluN1 subunit of NMDARs (Santa Cruz Biotechnology sc1467; $1: 250$ ) by following the procedure described above (see 'PA and EGFR immunoblottings' section).

Calcium video microscopy. Experiments were performed at room temperature on the stage of a Leica DMI6000B inverted microscope (Leica) equipped with a 150W Xenon high stability lamp and a Leica $\times 40,1.3$ numerical aperture epifluorescence oil immersion objective. Fura-2 ratio images were acquired with a Digital CMOS camera (Hamamatsu, Massy, France; ORCAFlash2.8 C11440-10C) and digitized (2048*2048) using the Metafluor 6.1 software (Universal Imaging Corporation, Downington, PA, USA). Cell cultures were transferred into a serum-free medium (HBBSS) and loaded with $10 \mu \mathrm{M}$ fura$2 \mathrm{AM}$ (Invitrogen) for $45 \mathrm{~min}$ at $37^{\circ} \mathrm{C}$. Neurons were washed, and NMDA treatment $(25 \mu \mathrm{M}$ for $30 \mathrm{~s})$ was applied using a peristaltic pump as baselines. Prior a second run of NMDA stimulations, neurons were incubated for 45 min with sc-tPA and tc-tPA alone or in the presence of either AG1478 (a blocker of the transphosphorylation of EGFRs) or an antibody targeting the N-terminal end of the GluN1 subunit of NMDA receptor (GluN1 antibody) previously characterized to prevent tPA-NMDAR interaction. ${ }^{23} \mathrm{~A}$ mean value of potentiation or inhibition was also measured, including all the recorded cells.

Excitotoxic neuronal death. Excitotoxicity was induced by exposure of cortical neurons to $50 \mu \mathrm{M}$ NMDA for $1 \mathrm{~h}$ or to $10 \mu \mathrm{M}$ of NMDA for $24 \mathrm{~h}$ in serum-free DMEM supplemented with $10 \mu \mathrm{M}$ of glycine at 12 DIV and performed as previously described..$^{25,31}$ The relative amount of IPA bound to cells was assessed by western blotting.

Induction of apoptosis. SD was induced by exposing neuronal cultures (7 DIV) to a serum-free DMEM supplemented with $10 \mu \mathrm{M}$ of glycine (+MK-801 at $10 \mu \mathrm{M}$ to prevent secondary exitototxicity) and characterized as previously described. ${ }^{31,32}$ The percentage of neuronal death was determined as the number of trypan blue-positive neurons after SD compared with the total number of neurons.

Statistical analysis. For calcium video microscopy with neurons (Figures 1,2), Shapiro test were used followed by Wilcoxon test to compare preincubation and postincubation responsiveness. Significance levels were defined as $\# P<0.0001$. In addition, for group comparison, Kruskal-Wallis tests were used, followed by Mann-Whitney U-tests as post-hoc tests. Significance levels were defined as ${ }^{*} P<0.0001$. Other statistical analyses were performed by the two-tailed Kruskall-Wallis' test, followed by post-hoc comparisons with the two-tailed Mann-Whitney's test.

\section{Conflict of Interest}

The authors declare no conflict of interest.
Acknowledgements. This work was supported by grants from the INSERM (French National Institute for Health and Medical Research), the University of CaenBasse Normandie, TC2N (Trans Channel Neuroscience Network) and the Regional Council of Lower Normandy. We thank Professor Carine Ali for review of this manuscript.

1. Thorsen $S$, Glas-Greenwalt $P$, Astrup T. Differences in the binding to fibrin of urokinase and tissue plasminogen activator. Thromb Diath Haemorrh 1972; 28: 65-74.

2. Polavarapu R, Gongora MC, Yi H, Ranganthan S, Lawrence D a, Strickland D et al. Tissue-type plasminogen activator-mediated shedding of astrocytic low-density lipoprotein receptor-related protein increases the permeability of the neurovascular unit. Blood 2007; 109: 3270-3278.

3. Tsirka SE, Rogove AD, Bugge TH, Degen JL, Strickland S. An extracellular proteolytic cascade promotes neuronal degeneration in the mouse hippocampus. J Neurosci 1997; 17: 543-552.

4. Shin CY, Kundel M, Wells DG. Rapid, activity-induced increase in tissue plasminogen activator is mediated by metabotropic glutamate receptor-dependent mRNA translation. J Neurosci 2004; 24: 9425-9433.

5. Gorter JA, Van Vliet EA, Rauwerda H, Breit T, Stad R, van Schaik L et al. Dynamic changes of proteases and protease inhibitors revealed by microarray analysis in CA3 and entorhinal cortex during epileptogenesis in the rat. Epilepsia 2007; 48: 53-64.

6. Rajapakse S, Ogiwara K, Takano N, Moriyama A, Takahashi T. Biochemical characterization of human kallikrein 8 and its possible involvement in the degradation of extracellular matrix proteins. FEBS Lett 2005; 579: 6879-6884.

7. Rijken DC, Hoylaerts M, Collen D. Fibrinolytic properties of one-chain and two-chain human extrinsic (tissue-type) plasminogen activator. J Biol Chem 1982; 257: 2920-2925.

8. Baranes $\mathrm{D}$, Lederfein $\mathrm{D}$, Huang $\mathrm{Y}$, Chen M, Bailey $\mathrm{CH}$, Kandel ER. Tissue plasminogen activator contributes to the late phase of LTP and to synaptic growth in the hippocampal mossy fiber pathway. Neuron 1998; 21: 813-825.

9. Liot G, Roussel BD, Lebeurrier N, Benchenane K, López-Atalaya JP, Vivien D et al. Tissue-type plasminogen activator rescues neurones from serum deprivation-induced apoptosis through a mechanism independent of its proteolytic activity. J Neurochem 2006; 98: 1458-1464.

10. Lee H-Y, Hwang I-Y, Im H, Koh J-Y, Kim Y-H. Non-proteolytic neurotrophic effects of tissue plasminogen activator on cultured mouse cerebrocortical neurons. J Neurochem 2007; 101: $1236-1247$.

11. Pittman RN, Ivins JK, Buettner HM. Neuronal plasminogen activators: cell surface binding sites and involvement in neurite outgrowth. J Neurosci 1989; 9: 4269-4286.

12. Seeds NW, Basham ME, Haffke SP. Neuronal migration is retarded in mice lacking the tissue plasminogen activator gene. Proc Nat. Acad Sci USA 1999; 96 : 14118-14123.

13. Kim Y. Nonproteolytic neuroprotection by human recombinant tissue plasminogen activator. Science 1999; 284: 647-650.

14. Henry VJ, Lecointre M, Laudenbach V, Ali C, Macrez R, Jullienne A et al. High t-PA release by neonate brain microvascular endothelial cells under glutamate exposure affects neuronal fate. Neurobiol Dis 2013; 50: 201-208.

15. Nicole O, Docagne F, Ali C, Margaill I, Carmeliet P, Mackenzie ET et al. The proteolytic activity of tissue-plasminogen activator enhances NMDA receptor-mediated signaling. Nat Med 2001; 7: 59-64.

16. Wang Y, Tsirka S, Strickland S, Stieg P, Soriano S, Lipton S. Tissue plasminogen activator (tPA) increases neuronal damage after focal cerebral ischemia in wild-type and tPA-deficient mice. Nat Med 1998; 4: 228-231.

17. Samson AL, Nevin ST, Croucher D, Niego B, Daniel PB, Weiss TW et al. Tissue-type plasminogen activator requires a co-receptor to enhance NMDA receptor function. J Neurochem 2008; 107: 1091-1101.

18. Parcq J, Bertrand T, Montagne A, Baron AF, Macrez R, Billard JM et al. Unveiling an exceptional zymogen: the single-chain form of TPA is a selective activator of NMDA receptordependent signaling and neurotoxicity. Cell Death Differ 2012; 19: 1983-1991.

19. Wu F, Echeverry R, Wu J, An J, Haile WB, Cooper DS et al. Tissue-type plasminogen activator protects neurons from excitotoxin-induced cell death via activation of the ERK1/2CREB-ATF3 signaling pathway. Mol Cell Neurosci 2013; 52: 9-19.

20. Wu F, Wu J, Nicholson AD, Echeverry R, Haile WB, Catano M et al. Tissue-type plasminogen activator regulates the neuronal uptake of glucose in the ischemic brain. $J$ Neurosci 2012; 32: 9848-9858.

21. Haile WB, Wu J, Echeverry R, Wu F, An J, Yepes M. Tissue-type plasminogen activator has a neuroprotective effect in the ischemic brain mediated by neuronal TNF- $\alpha$. J Cereb Blood Flow Metab 2012; 32: 57-69.

22. Correa F, Gauberti M, Parcq J, Macrez R, Hommet Y, Obiang P et al. Tissue plasminogen activator prevents white matter damage following stroke. J Exp Med 2011; 208: $1229-18$.

23. Macrez R, Obiang P, Gauberti M, Roussel B, Baron A, Parcq J et al. Antibodies preventing the interaction of tissue-type plasminogen activator with $\mathrm{N}$-methyl-D-aspartate receptors reduce stroke damages and extend the therapeutic window of thrombolysis. Stroke 2011; 42: 2315-2322. 
24. Liot G, Benchenane K, Léveillé F, López-Atalaya JP, Fernández-Monreal M, Ruocco A et al. 2,7-Bis-(4-amidinobenzylidene)-cycloheptan-1-one dihydrochloride, tPA stop, prevents tPA-enhanced excitotoxicity both in vitro and in vivo. J Cereb Blood Flow Metab 2004; 24 1153-1159.

25. Baron A, Montagne A, Cassé F, Launay S, Maubert E, Ali C et al. NR2D-containing NMDA receptors mediate tissue plasminogen activator-promoted neuronal excitotoxicity. Cell Death Differ 2010; 17: 860-871.

26. Rijken DC, Collen D. Purification and characterization of the plasminogen activator secreted by human melanoma cells in culture. J Biol Chem 1981; 256: 7035-7041.

27. Wallén $P$, Pohl $G$, Bergsdorf $N$, Rånby $M, N y T$, Jörnvall $H$. Purification and characterization of a melanoma cell plasminogen activator. Eur J Biochem 1983; 132: 681-686.

28. Reddrop C, Moldrich RX, Beart PM, Farso M, Liberatore GT, Howells DW et al. Vampire bat salivary plasminogen activator (desmoteplase) inhibits tissue-type plasminogen activatorinduced potentiation of excitotoxic injury. Stroke 2005; 36: 1241-1246.

29. Mantuano E, Lam MS, Gonias SL. LRP1 assembles unique co-receptor systems to initiate cell-signaling in response to tissue-type plasminogen activator and myelin-associated glycoprotein. J Biol Chem 2013; 288: 34009-34018.

30. Fleury V, Angles-Cano E. Characterization of the binding of plasminogen to fibrin surfaces: the role of carboxy-terminal lysines. Biochemistry 1991; 30: 7630-7638.
31. Nicole O, Ali C, Docagne F, Plawinski L, MacKenzie ET, Vivien D et al. Neuroprotection mediated by glial cell line-derived neurotrophic factor: involvement of a reduction of NMDA-induced calcium influx by the mitogen-activated protein kinase pathway. $J$ Neurosci 2001; 21: 3024-3033.

32. Koh JY, Gwag BJ, Lobner D, Choi DW. Potentiated necrosis of cultured cortical neurons by neurotrophins. Science 1995; 268: 573-575.

(c) Cell Death and Disease is an open-access journal published by Nature Publishing Group. This work is licensed under a Creative Commons Attribution 4.0 International License. The images or other third party material in this article are included in the article's Creative Commons license, unless indicated otherwise in the credit line; if the material is not included under the Creative Commons license, users will need to obtain permission from the license holder to reproduce the material. To view a copy of this license, visit http://creativecommons.org/licenses/by/4.0/

Supplementary Information accompanies this paper on Cell Death and Disease website (http://www.nature.com/cddis) 